\title{
The ambiguity function associated with the linear canonical transform
}

\author{
Tian-Wen Che, Bing-Zhao Li and Tian-Zhou Xu
}

\begin{abstract}
A new kind of ambiguity function (AF) associated with the linearcanonical transform (LCT) is proposed in this article, this new AF is defined based on the LCT and the classical AF. Firstly, the main properties and physical meanings of the newly defined AF are investigated, the results show that this kind of AF can be seen as one generalization of the classical AF. Then, the newly defined AF is applied to detect the parameters of the linear frequency modulated signals by combining with the classical Radon transform. The simulations are performed to verify the correctness of the derived results, and the comparison of the derived results with the common time-frequency analysis tools are also discussed.
\end{abstract}

Keywords: Linear canonical transform (LCT), Ambiguity function (AF), Radon transform (RT)

\section{Introduction}

Non-stationary signal processing and analysis is one of the most hottest research topics in signal processing community. A series of novel signal processing theories have been proposed to analyze the non-stationary signals, such as the short-time Fourier transform (STFT) [1], the wavelet transform (WT) [2] and the fractional Fourier transform (FRFT) [3,4]. As the generalization of the classical Fourier transform (FT), the FRFT has attracted more and more attention because of its inherent peculiarity, it is shown that the FRFT can be seen as a unified time-frequency transform [4]. The idea of the fractional Fourier operator is discussed in 1980 by Namias in quantum mechanics [5] and rediscovered in 1987 [6] in applied mathematics community. It was introduced into signal processing community by Almeida [7] and Santhanam and McClellan [8]. The discrete and digital computational methods of the FRFT proposed in $[9,10]$ open the door of its applications in real practical situations. As further generalization of the FRFT, the linear canonical transform (LCT) is shown to play an important role in optics and signal processing, many concepts associated with the FT have been generalized to the LCT domain. For example, the sampling theories [11,12], the eigenfunctions [13], the convolution theorem $[14,15]$, and the spectral analysis of

*Correspondence: li_bingzhao@bit.edu.cn

School of Mathematics, Beijing Institute of Technology, Beijing 100081, China uniform or nonuniform samples [16], are well studied in the LCT domain. The efficient discrete and digital computation algorithm of the LCT is introduced in $[17,18]$. More results associated with the LCT can be referred to $[3,4]$.

At the same time, the linear frequency-modulated (LFM) signal, a typical non-stationary signal, is widely used in communications, radar and sonar system. The LFM signal processing is so important that many algorithms and methods have been proposed. The ambiguity function (AF) associated with the FT is one of the most important time-frequency tools in LFM signal processing $[19,20]$. Besides, there are also proposed many other important and useful methods associated with the LFM signal parameter estimation and spectral analysis, such as the minimum mean square error (MMSE) estimation [21], the iterative algorithm [22], the generalized FT associate with the de Branges theory [23], the AF associated with the FRFT [24], the Wigner-Hough transform (WHT) [25], the Chirp FT [26], the Wiger-Ville distribution (WVD) [27] and the Radon-ambiguity transform (RAT) [28].

Following the classical definition of AF, Pei and Ding [29] firstly investigate the AF associated with the LCT, and obtains some important properties. Recently, Zhao et al. [30] investigates the properties and physical meaning of the AF associated with the LCT in optical signal processing community. Unlike the definition of AF in $[29,30]$, this article proposes a new kind of definition for AF associated

\section{Springer}

C 2012 Che et al; licensee Springer. This is an Open Access article distributed under the terms of the Creative Commons Attribution License (http://creativecommons.org/licenses/by/2.0), which permits unrestricted use, distribution, and reproduction in any medium, provided the original work is properly cited. 
with the LCT, the main properties and the application of the newly defined AF in the LFM signal processing are also discussed.

The article is organized as follows: In Section "Preliminary", we first review the related theory of the LCT, the classical AF, and the previous research outputs. In Section "Ambiguity function associated with the LCT", a new definition of AF associated with the LCT is proposed, and its main properties and physical meanings are introduced. The applications of the newly defined AF in the LFM signal processing is investigated in detail in Section "The applications of the AFL". Section "Discussions" discusses the comparison of the newly defined AF with the other common time-frequency tools, such as RWT, RAT, etc. Simulation results are given to show rationality and effectiveness of the proposed techniques in Section "Simulations". Section "Conclusion" is the conclusion.

\section{Preliminary}

The LCT

The LCT of a signal $f(t)$ can be defined as $[3,4]$

$$
\begin{aligned}
F_{(a, b, c, d)}(u) & =L^{(a, b, c, d)}[f(t)](u) \\
& = \begin{cases}\sqrt{\frac{1}{j 2 \pi b}} e^{j \frac{d}{2 b} u^{2}} \int_{-\infty}^{\infty} e^{j \frac{a}{2 b} t^{2}-j \frac{u t}{b}} f(t) d t & (b \neq 0) \\
\sqrt{d} e^{j \frac{c d}{2} u^{2}} f(d u) & (b=0)\end{cases}
\end{aligned}
$$

where $a, b, c, d$ are the real numbers and satisfying $a d-b c$ $=1$.

It is easy to verify that the classical FT, the FRFT, the chirp operation and the scaling operation are all special cases of the LCT as shown in the following.

When $(a, b, c, d)=(0,1,-1,0)$, the LCT becomes the FT:

$$
L^{(0,1,-1,0)}[f(t)](u)=\sqrt{-j} \operatorname{FT}[f(t)](u)
$$

When $(a, b, c, d)=(\cos \theta, \sin \theta,-\sin \theta, \cos \theta)$, the LCT becomes the FRFT:

$$
L^{(\cos \theta, \sin \theta,-\sin \theta, \cos \theta)}[f(t)](u)=\sqrt{e^{-j \alpha}} F^{\alpha}[f(t)](u)
$$

When $(a, b, c, d)=(1,0, \tau, 1)$, the LCT becomes the chirp operation:

$$
L^{(1,0, \tau, 1)}[f(t)](u)=e^{\frac{j}{2} \tau u^{2}} f(u)
$$

When $(a, b, c, d)=\left(\sigma, 0,0, \sigma^{-1}\right)$, the LCT becomes the scaling operation:

$$
L^{\left(\sigma, 0,0, \sigma^{-1}\right)}[f(t)](u)=\sqrt{\sigma^{-1}} f\left(\sigma^{-1} u\right)
$$

Many useful properties about the LCT $[3,4]$ show that the LCT is one of the most important non-stationary signal processing tools, the following additivity property and reversibility property associated with the LCT will be used this article.

(1) The additivity property

$$
L^{\left(a_{2}, b_{2}, c_{2}, d_{2}\right)}\left[L^{\left(a_{1}, b_{1}, c_{1}, d_{1}\right)}(f(t))\right](u)=L^{(a 3, b 3, c 3, d 3)}[f(t)](u)(6)
$$

where

$$
\left(\begin{array}{ll}
a_{3} & b_{3} \\
c_{3} & d_{3}
\end{array}\right)=\left(\begin{array}{ll}
a_{2} & b_{2} \\
c_{2} & d_{2}
\end{array}\right)\left(\begin{array}{ll}
a_{1} & b_{1} \\
c_{1} & d_{1}
\end{array}\right)
$$

(2) The reversibility property

$$
L^{(d,-b,-c, a)}\left[L^{(a, b, b, d)}[f(t)](u)\right](t)=f(t)
$$

Because the LCT can be looked as the generalization of the classical Fourier and fractional FT, so it can extends their utilities and applications and solves some problems that beyond those operations [31]. Without loss of generality, we only consider the case of $b \neq 0$ in this article, since the LCT is just a scaling transform operation when $b=0$. For more properties and the relations with the other transforms about the LCT, one can refer to $[3,4]$.

With the rapid developments of the modern signal processing technology, the classical concepts and theories in the FT domain have been studied extensively in the LCT domain, for example, the uniform and nonuniform sampling theories [32-34], the convolution and product theorems [14,15], the uncertainty principles [35-37] have been well studied and investigated in the LCT domain.

\section{Ambiguity function (AF)}

The instantaneous autocorrelation function of a signal $f(t)$ is defined as:

$$
R_{f}(t, \tau)=f\left(t+\frac{\tau}{2}\right) f^{*}\left(t-\frac{\tau}{2}\right)
$$

and the classical AF associated with the $\mathrm{FT}$ of $f(t)$ is defined as FT of $R_{f}(t, \tau)$ for $t$ :

$$
A F_{f}(\tau, u)=\int_{-\infty}^{\infty} R_{f}(t, \tau) e^{-j u t} d t
$$

or it can be equivalently defined in the FT domain as:

$$
A F_{f}(\tau, u)=\int_{-\infty}^{\infty} F\left(p+\frac{u}{2}\right) F^{*}\left(p-\frac{u}{2}\right) e^{j p \tau} d p
$$

where $F(p)$ is FT of the signal $f(t)$.

The properties of the classical AF can be listed in the following

$$
\begin{aligned}
& A F(\tau, u)=A F^{*}(-\tau,-u) \\
& A F(0,0)=\int_{-\infty}^{\infty}|f(t)|^{2} d t
\end{aligned}
$$




$$
\frac{1}{2 \pi} \int_{-\infty}^{\infty} \int_{-\infty}^{\infty} A F_{x}(\tau, u) A F_{z}(\tau, u) d \tau d u=|<x, z>|^{2}
$$

The AF is one of the classical and important timefrequency signal processing tools. It is shown in [28] that the AF of a LFM signal passes through the origin of ambiguity plane $(\tau, u)$, and the slope of the projection line is the frequency rate of the signal. All of these properties of the AF can help us to obtain good results in the LFM signal processing and the parameter estimation.

\section{Prior research outputs}

Based on the properties of the LCT and the definition of the AF associated with the FT, Pei and Ding [29] and Zhao et al. [30] proposed two different definitions of AF associated with the LCT in the following.

Definition 1 : Suppose the LCT of a signal $f(t)$ with parameter $(a, b, c, d)$ is $F_{(a, b, c, d)}(u)$, then the AF associated with the LCT is defined as [29]

$$
\begin{aligned}
A F_{\left.F_{(} a, b, c, d\right)}(\tau, u)= & \int_{-\infty}^{\infty} F_{(a, b, c, d)}\left(v+\frac{\tau}{2}\right) F_{(a, b, c, d)}^{*} \\
& \times\left(v-\frac{\tau}{2}\right) e^{-j u v} d v
\end{aligned}
$$

It is shown in [29] that this kind of definition of AF has the following properties

$$
\begin{array}{r}
A F_{\left.F_{(} a, b, c, d\right)}(\tau, u)=A F(d \tau-b u,-c \tau+a u) \\
A F_{\left.F_{(} a, b, c, d\right)}(a \tau+b u, c \tau+d u)=A F(\tau, u)
\end{array}
$$

where $A F(\tau, u)$ and $A F_{F}(\tau, u)$ represent the $\operatorname{AF}$ of $f(t)$ and $F_{(a, b, c, d)}(v)$, respectively.

Definition 2 : Suppose the LCT of a signal $f(t)$ with parameter $(a, b, c, d)$ is $F_{(a, b, c, d)}(v)$, then the linear canonical AF (LCAF) is defined as [30]

$$
\begin{aligned}
A F_{M}(\tau, u)= & \int_{-\infty}^{\infty} F_{(a, b, c, d)}\left(v+\frac{\tau}{2}\right) F_{(a, b, c, d)}^{*} \\
& \times\left(v-\frac{\tau}{2}\right) e^{j u v} d v
\end{aligned}
$$

It is shown in [30] that this kind of definition of AF has the following properties

$$
\begin{aligned}
& A F_{M}(\tau, u)=A F(b \tau+d u,-a \tau-c u) \\
& A F_{M}(-u, \tau)=A F(d \tau-b u,-c \tau+a u)
\end{aligned}
$$

The other properties and physical meanings of those kinds of AF associated with the LCT, are also investigated in detail in $[29,30]$.

It is easy to see that the generalized AF defined by Equations (14) and (17) are the FT of the instantaneous autocorrelation function of LCT signal. They are actually the linear coordinate transformation of traditional AF in the FT domain. Unlike the definitions in $[29,30]$, we define a new kind of AF associated with the LCT in this article, and the applications of the newly defined AF in LFM signal processing are also investigated in the following sections.

\section{AF associated with the LCT}

The new definition of AF associated with the LCT

From the mathematical view point, many transforms in signal processing community can be seen as the product of a signal with a kernel function. They can be also classified into two kind of definitions: (1) the product of the local form of a signal with a kernel function, such as STFT, WVD, etc; (2) the product of the signal and a local kernel function, such as WT, Gabor transform, etc.

It is easy to see that the generalized AF in $[29,30]$ is based on the local transform of original signal. The LCT of original signal is firstly obtained, and then applied the traditional AF definition, which can be considered as the first kind of definition. Unlike their definitions, we propose a new definition of the AF associated with the LCT following the second kind of definition.

Definition 3: The AF of a signal $f(t)$ associated with the LCT with parameter $A=(a, b, c, d)$ is defined as

$$
\begin{aligned}
\operatorname{AFL}[f(t)](\tau, u) & =A F_{(a, b, c, d)}(\tau, u) \\
& =\int_{-\infty}^{\infty} R_{f}(t, \tau) K_{A}(t, u) d t
\end{aligned}
$$

where $K_{A}(t, u)=\sqrt{\frac{1}{j 2 \pi b}} e^{j \frac{d}{2 b} u^{2}} e^{j \frac{a}{2 b} t^{2}-j \frac{u t}{b}}$ and $R_{f}(t, \tau)=$ $f\left(t+\frac{\tau}{2}\right) f^{*}\left(t-\frac{\tau}{2}\right)$.

From the definition and the physical meaning of the LCT $[3,4]$, this kind of AF can be interpreted as the affine transform of the instantaneous autocorrelation function $R_{f}(t, \tau)$ in the $(\tau, u)$ plane. In order to make it different from the existing definition associated with the LCT, we denote the AF of a signal $f(t)$ defined by Equation (20) with $A F_{(a, b, c, d)}(\tau, u)$, and simplified as AFL, in the following sections.

\section{The properties of newly defined AFL}

If the AFL of a signal $f(t)$ is denotes as $A F_{(a, b, c, d)}(\tau, u)$, then it is easy to show that the AFL has the following properties.

\section{Conjugation property}

The AFL of $f^{*}(t)$ is $\left[A F_{(a,-b,-c, d)}(\tau, u)\right]^{*}$, the AFL of $f(-t)$ is $\left[A F_{(a,-b,-c, d)}(\tau,-u)\right]^{*}$; and the AFL of $f^{*}(-t)$ is $A F_{(a, b, c, d)}(\tau,-u)$.

\section{Shifting property}

The AFL of $f(t-p)$ is $e^{-j \frac{a c}{2} p^{2}+j c p u} A F_{(a, b, c, d)}(\tau, u-p a)$, the AFL of $f(t) e^{j w t}$ is $e^{j w \tau} A F_{(a, b, c, d)}(\tau, u)$, and the AFL of $f(t) e^{j w t^{2}}$ is $e^{-j 2 d w^{2} \tau^{2} b+j 2 d u w \tau} A F_{(a, b, c, d)}(\tau, u-2 w \tau b)$. 


\section{Limited support}

Iff $(t)=0, t \notin\left[t_{1}, t_{2}\right]$, then $A F_{(a, b, c, d)}(\tau, u)=0, \tau>t_{2}-t_{1}$.

\section{Anti-derivative property}

The signal $f(t)$ can be expressed by the AFL of $f(t)$ by the following formula:

$$
f(t)=\frac{1}{f^{*}(0)} \int_{-\infty}^{\infty} A F_{(a, b, c, d)}(t, u) K_{A^{-1}}\left(\frac{t}{2}, u\right) d u
$$

\section{Special cases}

when $(a, b, c, d)=(0,1,-1,0)$, the AFL becomes the classical AF [19],

$$
A F_{(0,1,-1,0)}(\tau, u)=\sqrt{-j} A F(\tau, u),
$$

and when $(a, b, c, d)=(\cos \theta, \sin \theta,-\sin \theta, \cos \theta)$, the AFL becomes the AF associated with the FRFT $\left(A F^{\alpha}(\tau, u)\right)$,

$$
A F_{(\cos \theta, \sin \theta,-\sin \theta, \cos \theta)}(\tau, u)=\sqrt{e^{-j \alpha}} A F^{\alpha}(\tau, u) .
$$

\section{Energy in time-domain}

$$
\int_{-\infty}^{\infty}|f(t)|^{2} d t=\sqrt{j 2 \pi} A F_{(0,1,-1,0)}(0,0) .
$$

\section{Symmetry}

$\left|A F_{(a, b, c, d)}(-\tau,-u)\right|=\left|A F_{(-a, b, c,-d)}(\tau, u)\right|$.

\section{Sensitivity for LFM signal}

The AFL of a LFM signal $f(t)=e^{j\left(w_{0} t+m_{0} t^{2}\right)}$ is

$A F_{(a, b, c, d)}(\tau, u)=\left\{\begin{array}{l}e^{j\left(\frac{d}{2 b} u^{2}+w_{0} \tau\right)} \sqrt{\frac{2 \pi|b|}{j}} \delta\left(u-m_{0} \tau b\right)\left(\frac{a}{2 b}=0\right) \\ e^{j\left(\frac{d}{2 b} u^{2}+w_{0} \tau-\frac{\left(m_{0} \tau-u / b\right)^{2}}{2 a / b}\right)} \sqrt{\frac{1}{a}}\left(\frac{a}{2 b} \neq 0\right)\end{array}\right.$

where $w_{0}, m_{0}$ represent the initial frequency and frequency rate of $f(t)$, respectively.

Proof. From the definition of the AFL, we obtain

$$
\begin{aligned}
A F_{(a, b, c, d)}(\tau, u) & =\sqrt{\frac{1}{j 2 \pi b}} e^{j \frac{d}{2 b} u^{2}} \int_{-\infty}^{\infty} e^{j \frac{a}{2 b} t^{2}-j \frac{u t}{b}} R_{f}(t, \tau) d t \\
& =\sqrt{\frac{1}{j 2 \pi b}} e^{j \frac{d}{2 b} u^{2}} \int_{-\infty}^{\infty} e^{j \frac{a}{2 b} t^{2}-j \frac{u t}{b}} e^{j\left(a_{1} \tau+2 a_{2} \tau t\right)} d t \\
& =\sqrt{\frac{1}{j 2 \pi b}} e^{j\left(\frac{d}{2 b} u^{2}+a_{1} \tau\right)} \int_{-\infty}^{\infty} e^{j \frac{a}{2 b} t^{2}} e^{j\left(2 a_{2} \tau-\frac{u}{b}\right) t} d t \\
\frac{a}{2 b} & =0,=\sqrt{\frac{1}{j 2 \pi b}} e^{j\left(\frac{d}{2 b} u^{2}+a_{1} \tau\right) \int_{-\infty}^{\infty} e^{j\left(2 a_{2} \tau-\frac{u}{b}\right) t} d t} \\
& =\sqrt{\frac{2 \pi b}{j}} e^{j\left(\frac{d}{2 b} u^{2}+a_{1} \tau\right)} \delta\left(u-2 a_{2} \tau\right)
\end{aligned}
$$

$$
\begin{aligned}
\frac{a}{2 b} \neq 0,= & \sqrt{\frac{1}{j 2 \pi b}} e^{j\left(\frac{d}{2 b} u^{2}+a_{1} \tau\right)} \int_{-\infty}^{\infty} e^{j \frac{a}{2 b} t^{2}} e^{j\left(2 a_{2} \tau-\frac{u}{b}\right) t} d t \\
= & \sqrt{\frac{1}{j 2 \pi b}} e^{j\left(\frac{d}{2 b} u^{2}+a_{1} \tau\right)} \int_{-\infty}^{\infty} e^{j \frac{a}{2 b}\left(t^{2}+\frac{2 a_{2} \tau-u / b}{a / 2 b}\right)} d t \\
= & \sqrt{\frac{1}{j 2 \pi b}} e^{j\left(\frac{d}{2 b} u^{2}+w_{0} \tau\right)} e^{-j \frac{a}{2 b} \frac{\left(m_{0} \tau-u / b\right)^{2}}{a^{2} / b^{2}}} \\
& \times \int_{-\infty}^{\infty} e^{j \frac{a}{2 b}\left(t+\frac{m_{0} \tau-u / b}{a / b}\right)^{2}} d t \\
= & \sqrt{\frac{1}{a}} e^{j\left(\frac{d}{2 b} u+a_{1} \tau\right)} e^{-j \frac{a}{2 b} \frac{\left(2 a_{2} \tau-u / b\right)^{2}}{a^{2} / b^{2}}}
\end{aligned}
$$

We can see from Equation (21) that the AFL of $f(t)$ will produces one impulse in $(\tau, u)$ plane when the parameters meet the special condition. There will be also one crest because of energy accumulation when the signal is finite. The AFL of the LFM signal will pass through the origin of ambiguity plane $(\tau, u)$, and the slope of projection line is $b$ times of the frequency rate.

The relationship between the AFL and STFT

Suppose the STFT of signal $f(t)$ is defined as

$$
\operatorname{STFT}^{w}(t, u)=\int_{-\infty}^{\infty} f(p) w^{*}(p-t) e^{-j u p} d p .
$$

Then the following Lemma 1 reflects the relationship between the AFL and the STFT of a signal $f(t)$.

Lemma 1. The AFL of a signal $f(t)$ with parameter $(a, b, c, d)$ can be seen as the STFT of signal $f(t)$ with $w(t)=f(t) e^{j \frac{a}{2 b} t^{2}}$.

$$
\begin{aligned}
A F_{(a, b, c, d)}(\tau, u)= & \sqrt{\frac{1}{j 2 \pi b}} e^{j\left[\frac{d}{2 b} u^{2}-\frac{a}{2 b} \frac{3 \tau^{2}}{4}+\frac{u \tau}{2 b}\right]} \operatorname{STFT}^{w} \\
& \times\left(\tau, \frac{2 u-a \tau}{2 b}\right)
\end{aligned}
$$

Proof.

$$
\begin{aligned}
\operatorname{AF}_{(a, b, c, d)}(\tau, u)= & \sqrt{\frac{1}{j 2 \pi b}} e^{j \frac{d}{2 b} u^{2}} \int_{-\infty}^{\infty} f\left(t+\frac{\tau}{2}\right) f^{*} \\
& \times\left(t-\frac{\tau}{2}\right) e^{j \frac{a}{2 b} t^{2}-j \frac{u t}{b}} d t \\
= & \sqrt{\frac{1}{j 2 \pi b}} e^{j \frac{d}{2 b} u^{2}} \int_{-\infty}^{\infty} f(p) f^{*} \\
& \times(p-\tau) e^{j \frac{a}{2 b}(p-\tau+\tau / 2)^{2}-j \frac{u(p-\tau / 2)}{b}} d p \\
= & \sqrt{\frac{1}{j 2 \pi b}} e^{j\left[\frac{d}{2 b} u^{2}-\frac{a}{2 b} \frac{3 \tau^{2}}{4}+\frac{u \tau}{2 b}\right]} \int_{-\infty}^{\infty} f(p) f^{*} \\
& \times(p-\tau) e^{j \frac{a}{2 b}(p-\tau)^{2}} e^{j\left(\frac{a}{2 b} p \tau-\frac{u p}{b}\right)} d p
\end{aligned}
$$




$$
\begin{aligned}
= & \sqrt{\frac{1}{j 2 \pi b}} e^{j\left[\frac{d}{2 b} u^{2}-\frac{a}{2 b} \frac{3 \tau^{2}}{4}+\frac{u \tau}{2 b}\right]} \int_{-\infty}^{\infty} f(p) f^{*} \\
& \times(p-\tau) e^{j \frac{a}{2 b}(p-\tau)^{2}} e^{-j\left(\frac{u}{b}-\frac{a \tau}{2 b}\right) p} d p \\
= & \sqrt{\frac{1}{j 2 \pi b}} e^{j\left[\frac{d}{2 b} u^{2}-\frac{a}{2 b} \frac{3 \tau^{2}}{4}+\frac{u \tau}{2 b}\right]} \operatorname{STFT}^{w} \\
& \times\left(\tau, \frac{2 u-a \tau}{2 b}\right)
\end{aligned}
$$

\section{The applications of the AFL}

In order to show the effective of the newly defined AFL in the LFM signal detection, we will apply the AFL to the LFM signal analysis and parameter detection in this section. Following with the classical LFM signal detection methods [26-28], the detector proposed in this article is obtained by combining the newly defined AFL with Radon transform (RT), in order to make this method different from the traditional methods, it is denoted as "the AFL combined with RT", and simplified as RAFL.

\section{The detector}

The RT, which is commonly used for the reconstruction of images in computer tomography, is defined as

$$
R_{s, \varphi}(f(x, y))=\int_{-\infty}^{\infty} \int_{-\infty}^{\infty} f(x, y) \delta(x \sin \varphi+y \cos \varphi-s) d x d y
$$

for $-\infty<s<\infty,-\pi / 2<\varphi<\pi / 2$, where the delta function specifies the direction of integration, $s$ is the distance between the origin and a line in the $x-y$ plane, $\varphi$ is the angle between the line and the $x$ axis.

From the analysis of the AFL of a LFM signal, we know that the direction of interest passes through the origin of the ambiguity plane, if we set the parameter of the RT with $s=0$ to the phase-free AFL of Equation (20), then the detection of signal can be reduced from the 2-D search problem to a 1-D search problem. As is known from Equation (21), the slope value of AFL is $b$ times of chirp rate of LFM signal, it is therefore essentially to compute the line integral along a straight line with its direction specified by the delta function $\delta(u-b m \tau)$ in the ambiguity plane in order to realize energy accumulation.

In [27], the author uses the squared-modulus of the AF as the function to which applies the RT to be the detector. Along with this idea, we use the squared-modulus of the AFL as the function to which applies the RT to be the detector in our article. Following the discussion above, the detector associated with the newly defined AFL is defined in the following form:

$$
\begin{aligned}
\eta(m) & =\int_{-\infty}^{\infty} \int_{-\infty}^{\infty}\left|A F_{(a, b, c, d)}(\tau, u)\right|^{2} \delta(u-b m \tau) d \tau d u \\
& =\int_{-\infty}^{\infty}\left|A F_{(a, b, c, d)}(\tau, b m \tau)\right|^{2} d \tau
\end{aligned}
$$

The detector $\eta(m)$ can be used to realize the energy accumulation, from Equation (21)

$$
\begin{aligned}
\frac{a}{2 b}= & 0, \eta(m)=\int_{-\infty}^{\infty} \int_{-\infty}^{\infty}\left|\sqrt{\frac{2 \pi b}{j}} e^{j\left(\frac{d}{2 b} u^{2}+w_{0} \tau\right)} \delta\left(u-m_{0} \tau\right)\right|^{2} \delta \\
& \times(u-b m \tau) d \tau d u \\
= & |2 \pi b| \int_{-\infty}^{\infty}\left|e^{j\left(\frac{b d}{2} m^{2} \tau^{2}+w_{0} \tau\right)} \delta\left(b m \tau-b m_{0} \tau\right)\right|^{2} d \tau
\end{aligned}
$$

$$
\begin{aligned}
\frac{a}{2 b} & \neq 0, \eta(m)=\int_{-\infty}^{\infty} \int_{-\infty}^{\infty}\left|\sqrt{\frac{1}{a}} e^{j\left(\frac{d}{2 b} u^{2}+w_{0} \tau\right)} e^{-j \frac{a}{2 b} \frac{\left(m_{0} \tau-u / b\right)^{2}}{a^{2} / b^{2}}}\right|^{2} \delta \\
& \times(u-b m \tau) d \tau d u \\
= & \left|\frac{1}{a}\right| \int_{-\infty}^{\infty}\left|e^{j\left(\frac{b c m+m_{0}}{2 a} b m\right.} e^{j \frac{b}{2 a}\left(m-m_{0}\right) m_{0}+w_{0} \tau}\right|^{2} d \tau
\end{aligned}
$$

From Equations (24) and (25), we can see that $\eta(m) \rightarrow \infty$ when $m \rightarrow m_{0}$. Therefore, the signal can be detected by calculating $\eta(m)$ and comparing it with the threshold.

\section{Detection of LFM signal \\ One component LFM}

Suppose the signal $f(t)$ is modeled as following and has unit energy with time duration $T$ :

$$
f(t)=\frac{1}{\sqrt{T}} e^{j\left(w_{0} t+\frac{m_{0}}{2} t^{2}\right)} \quad\left(|t|<\frac{T}{2}\right)
$$

We can obtain the modulus of AFL of $f(t)$ by the definition of AFL

$\left|A F_{(a, b, c, d)}(\tau, u)\right|=\left\{\begin{array}{l}\frac{\sin \left[\left(u / b-m_{0} \tau\right) T / 2\right]}{\left(u / b-m_{0} \tau\right) T / 2} \sqrt{\frac{1}{2 \pi b}} \quad(a=0) \\ \left|\sqrt{\frac{1}{2 \pi b}} \int_{-T / 2}^{T / 2} e^{j \frac{a}{2 b} t^{2}+j\left(m_{0} \tau-u / b\right) t} d t\right|(a \neq 0)\end{array}\right.$

When $a=0$, we can obtain the analytic solution of detector $\eta(m)$,

$$
\begin{aligned}
\eta(m) & =\frac{1}{T} \int_{-\infty}^{\infty} \int_{-\infty}^{\infty}\left|A F_{(a, b, c, d)}(\tau, u)\right|^{2} \delta(u-b m \tau) d \tau d u \\
& =\frac{2}{T^{3}} \int\left|\frac{\sin \left[\left(m-m_{0}\right) \tau T / 2\right]}{\left(m-m_{0}\right) \tau / 2} \sqrt{\frac{j}{2 \pi b}}\right|^{2} d \tau
\end{aligned}
$$

The coefficient in front of the integral in Equation (27) is added for normalization purposes. When $m=m_{0}$, Equation (27) yields

$$
\eta\left(m_{0}\right)=\frac{2}{T} \int_{0}^{T} \frac{1}{2 \pi b} d \tau=\frac{1}{\pi b}
$$


For $m>m_{0}$, let $\left(m-m_{0}\right) / 2=p$, so that Equation (27) is expressed as

$$
\begin{aligned}
\eta\left(2 p+m_{0}\right)= & \frac{1}{2 \pi b} \frac{2}{T^{3}} \int_{0}^{T} \frac{\sin ^{2}[p \tau T]}{(p \tau)^{2}} d \tau \\
= & \frac{1}{2 \pi b}\left\{\frac{2}{p T^{2}} \int_{0}^{\frac{p T^{2}}{2}} \frac{\sin ^{2}(x)}{x^{2}} d x\right. \\
& \left.+\frac{2}{T^{3}} \int_{T / 2}^{T} \frac{\sin ^{2}(p \tau T)}{(p \tau)^{2}} d \tau\right\}
\end{aligned}
$$

It shown in [38] that the following identities hold,

$$
\int \frac{\sin ^{2}(x)}{x^{2}} d x=\operatorname{si}(2 x)-\frac{\sin ^{2}(x)}{x}
$$

where

$$
s i(x)=\int_{0}^{x} \frac{\sin (t)}{t} d t .
$$

Substitute Equation (30) into Equation (29) and use the following inequality

$$
\int_{T / 2}^{T} \frac{\sin ^{2}(p \tau T)}{(p \tau)^{2}} d \tau<\frac{1}{p^{2}} \int_{T / 2}^{T} \frac{1}{\tau^{2}} d \tau=\frac{1}{p^{2} T}
$$

the Equation (29) can be rewritten

$\eta\left(2 p+m_{0}\right)<\frac{1}{2 \pi b}\left\{\frac{2}{p T^{2}}\left[s i\left(p T^{2}\right)-\frac{2}{p T^{2}} \sin ^{2}\left(\frac{p T^{2}}{2}\right)+\frac{1}{p T^{2}}\right]\right\}$.

By letting $2 p+m_{0}=m$, we obtain

$$
\begin{aligned}
\eta(m)< & \frac{2}{\pi b\left(m-m_{0}\right) T^{2}}\left[s i\left(\frac{\left(m-m_{0}\right) T^{2}}{2}\right)+\frac{2}{\left(m-m_{0}\right) T^{2}} \cos \right. \\
& \left.\times\left(\frac{\left(m-m_{0}\right) T^{2}}{2}\right)\right]
\end{aligned}
$$

Thus, the detector $\eta(m)$ of one component signal has been derived, and Equation (31) gives the upper bound of the detector for $m \geq m_{0}$, and the $\eta\left(m+m_{0}\right)$ can be estimated by $\eta(m)=\eta\left(2 m_{0}-m\right)$ for $m<m_{0}$. However, when $a \neq 0$, we can no longer expect an analytic solution to $\eta(m)$, the numerical method has to be used.

Based on these results, we can detect the LFM signal by calculating the detector $\eta(m)$. The proposed detector yields maxima over chirp rates of the LFM signal. When $T$ is finite, $\eta(m)$ has one crest, otherwise $\eta(m)$ becomes delta function as discussed before.

\section{Multicomponent LFM}

The bicomponent signal is modeled as

$$
f(t)= \begin{cases}\frac{1}{\sqrt{T}} e^{j\left(w_{0} t+\frac{m_{0}}{2} t^{2}\right)}+\frac{1}{\sqrt{T}} e^{j\left(w_{1} t+\frac{m_{1}}{2} t^{2}\right)} & \left(|t|<\frac{T}{2}\right) \\ 0 & \left(|t|>\frac{T}{2}\right)\end{cases}
$$

with the assumption that $w_{0}=w_{1}$ and $m_{0}>m_{1}$ for simplicity purpose. The AFL of $f(t)$ can be derived as

$$
A F_{(a, b, c, d)}(\tau, u)=\left\{\begin{array}{rr}
\frac{\sin \left[\left(m_{0} \tau-u / b\right) T / 2\right]}{\left(m_{0} \tau-u / b\right) T / 2} \sqrt{\frac{j}{2 \pi b}}+\frac{\sin \left[\left(m_{1} \tau-u / b\right) T / 2\right]}{\left(m_{1} \tau-u / b\right) T / 2} \\
\quad \times \sqrt{\frac{j}{2 \pi b}}+\frac{2}{T} \sqrt{\frac{j}{2 \pi b} \sqrt{\frac{\pi}{m_{0}-m_{1}}}} & (|\tau| \leq T) \\
\left\{\left[C\left(X_{0}\right)+C\left(X_{1}\right)\right] \cos \left(a_{m} \tau^{2}\right)\right. & \\
\left.+\left[S\left(X_{0}\right)+S\left(X_{1}\right)\right] \sin \left(a_{m} \tau^{2}\right)\right\} & (a=0) \\
0 & (|\tau|>T)
\end{array}\right.
$$

where $C(x)=\int_{0}^{x} \cos \left(\frac{\pi t^{2}}{2}\right) d t$ and $S(x)=\int_{0}^{x} \sin \left(\frac{\pi t^{2}}{2}\right) d t$ are Fresnel integrals, and other parameters, such as $X_{0}, X_{1}$ are the same as in [28].

The first two terms in Equation (32) represent the auto terms of the signal, whereas the rest is the cross term. When $a \neq 0$, we can not get the analytic solution to AFL. Similar with the situation in [28], we can no longer expect an analytic solution of $\eta(m)$ because the equation of AFL is rather complex, for example, including the $C\left(X_{0}\right), C\left(X_{1}\right), S\left(X_{0}\right), S\left(X_{1}\right)$. In the simulation section, we will give the performance of detector by the numerical method to verify the detection of LFM signal.

\section{Detection of quadratic FM (QFM) signal}

Non-linear FM signal is applied widely in nature and human applications. For example, cubic and quadratic FM signal are applied to locate echoes in the bat's sonar system. Since nonlinear FM signals are used in various subjects, it is essential to investigate them by the newly defined AFL.

The QFM signal is defined as $f(t)=A e^{j\left(a_{1} t+a_{1} t^{2}+a_{3} t^{3}\right)}$, and the AFL of $f(t)$ is obtained as

$$
A F_{(a, b, c, d)}(\tau, u)=\left\{\begin{array}{l}
\sqrt{\frac{2 \pi|b|}{j}} A^{2} e^{j\left(\frac{d}{2 b} u^{2}+a_{1} \tau+\frac{a_{3}}{4} \tau^{3}\right)} \delta\left(u-2 a_{2} \tau b\right) \\
\times\left(3 a_{3} \tau+\frac{a}{2 b}=0\right) \\
\sqrt{\frac{1}{6 a_{3} b \tau+a}} A^{2} e^{j\left(\frac{d}{2 b} u^{2}+a_{1} \tau+\frac{a_{3}}{4} \tau^{3}\right)-j \frac{\left(2 a_{2} \tau-u / b\right)^{2}}{2\left(6 a_{3} \tau+a / b\right)}} \\
\times\left(3 a_{3} \tau+\frac{a}{2 b} \neq 0\right)
\end{array}\right.
$$

Proof.

$$
\begin{aligned}
A F_{(a, b, c, d)}(\tau, u)= & \sqrt{\frac{1}{j 2 \pi b}} A^{2} e^{j \frac{d}{2 b} u^{2}} \int_{-\infty}^{\infty} e^{j\left[a_{1} \tau+\frac{a_{3} \tau^{3}}{4}+2 a_{2} \tau t+3 a_{3} \tau t^{2}\right]} \\
& \times e^{j \frac{a}{2 b} t^{2}-j \frac{u t}{b}} d t=\sqrt{\frac{1}{j 2 \pi b}} A^{2} e^{j \frac{d}{2 b} u^{2}} \\
& \times \int_{-\infty}^{\infty} e^{j\left(3 a_{3} \tau+\frac{a}{2 b}\right) t^{2}+j\left(2 a_{2} \tau-\frac{u}{b}\right) t+j\left(a_{1} \tau+a_{3} \frac{\tau^{3}}{4}\right)} d t
\end{aligned}
$$




$$
\begin{aligned}
& 3 a_{3} \tau+\frac{a}{2 b}=0, A F_{(a, b, c, d)}(\tau, u)=\sqrt{\frac{1}{j 2 \pi b}} A^{2} e^{j \frac{d}{2 b} u^{2}} \\
& \times \int_{-\infty}^{\infty} e^{j\left[\left(2 a_{2} \tau-\frac{u}{b}\right) \tau+\left(a_{1} \tau+a_{3} \frac{\tau^{3}}{4}\right)\right]} d t \\
& =\sqrt{\frac{1}{j 2 \pi b}} A^{2} e^{j \frac{d}{2 b} u^{2}+j\left(a_{1} \tau+a_{3} \frac{\tau^{3}}{4}\right)} \int_{-\infty}^{\infty} e^{j\left[\left(2 a_{2} \tau-\frac{u}{b}\right) \tau\right.} d t \\
& =\sqrt{\frac{2 \pi b}{j}} A^{2} e^{j\left(\frac{d}{2 b} u^{2}+a_{1} \tau+a_{3} \frac{\tau^{3}}{4}\right)} \delta\left(u-2 a_{2} b \tau\right) \\
& 3 a_{3} \tau+\frac{a}{2 b} \neq 0, A F_{(a, b, c, d)}(\tau, u)=\sqrt{\frac{1}{j 2 \pi b}} A^{2 j\left(\frac{d}{2 b} u^{2}+a_{1} \tau+a_{3} \frac{\tau^{3}}{4}\right)} \\
& \times \int_{-\infty}^{\infty} e^{j\left(3 a_{3} \tau+\frac{a}{2 b}\right)\left(t^{2}+\frac{\left(2 a_{2} \tau-u / b\right)}{3 a_{3} \tau+\frac{a}{2 b}}\right)} d t \\
& =\sqrt{\frac{1}{j 2 \pi b}} A^{2} e^{j\left(\frac{d}{2 b} u^{2}+a_{1} \tau+a_{3} \frac{\tau^{3}}{4}\right)-j \frac{\left(2 a_{2} \tau-u / b\right)^{2}}{2\left(6 a_{3} \tau+\frac{a}{b}\right)}} \\
& \times \int_{-\infty}^{\infty} e^{j\left(3 a_{3} \tau+\frac{a}{2 b}\right)\left(t+\frac{\left(2 a_{2} \tau-u / b\right)}{3 a_{3} \tau+\frac{a}{2 b}}\right)^{2}} d t \\
& =\sqrt{\frac{1}{a+6 a_{3} \tau b}} A^{2} e^{j\left(\frac{d}{2 b} u^{2}+a_{1} \tau+a_{3} \frac{\tau^{3}}{4}\right)-j \frac{\left(2 a_{2} \tau-u / b\right)^{2}}{2\left(6 a_{3} \tau+\frac{a}{b}\right)}}
\end{aligned}
$$

From Equation (33), when we choose the special parameters satisfying $3 a_{3} \tau+\frac{a}{2 b}=0$, the AFL of QFM signal can generate one impulse in $(\tau, u)$ plane. Similar with the discussion of the $\eta(m)$ for multicomponent LFM signal, we can not obtain the analytic solution for this situation.

In fact, the cubic phase parameter can be seen as the information that we want to obtain, and the other phase parameter can been as the noise in detection of signal. It is concluded from the Equation (33) that the AFL has the time-frequency focus, therefore the cubic phase information of the QFM signal can be detected by this way. We will apply the numerical methods in the simulation sections about the detector of this kind of signal.

\section{Discussions}

It is shown in previous section that the AFL can be used to the detection of the LFM signals, and the AFL combined with RT (RAFL) is shown to be effective in LFM signal detection in previous sections. In this section, we will compare the RAFL with other common time-frequency methods, including the RAT, the Radon-WVD transform (RWT), the WVD-Hough transform (WHT). Firstly, we show that the RAT and RWT can be seen as equivalent, then we compare the RAFL with RAT, WHT, FRFT in the following part.

\section{The RAT and Radon-WVD transform (RWT)}

The WVD of a LFM signal $f(t)=e^{j\left(w_{0} t+m t^{2} / 2\right)}$ is given by

$$
\operatorname{WVD}(t, w)=\delta\left(w-w_{0}-m t\right)
$$

It can be seen from Equation (34) that the WVD of one component LFM signal is an impulse spectrum along straight line $w=w_{0}-m t$. In other words, WVD has the satisfying time-frequency focus. However, the WVD of multicomponent LFM signal will produces cross terms, which will blurs time-frequency plane, especially in low SNR environment. The Radon-WVD (RWT) is the integration of the WVD over all possible lines in the time-frequency plane, which can transform the signal detection problem to the 2-D search problem.

The AF of $f(t)$ in the classical FT sense is given by

$$
A F(\tau, u)=e^{j w_{0} \tau} \delta(u-m \tau)
$$

The AF also passes through the origin of the ambiguity plane and satisfies the time-frequency focus property. The line integral of the RT is performed over all lines passing through the origin of the ambiguity plane to yield the RAT. The RAT reduces from the 2-D search problem to the 1-D search problem [28]. It is obviously that the AF and WVD of a signal is the 2-D FT pair, and AF can be obtained by applying 2-D FT to WVD.

Based on discussions above, the RWT and RAT are equivalent in the LFM signal detection.

\section{The RAFL and RAT}

The AF and AFL of a signal $f(t)$ are

$$
\begin{aligned}
& A F(\tau, u)=\int_{-\infty}^{\infty} f\left(t+\frac{\tau}{2}\right) f^{*}\left(t-\frac{\tau}{2}\right) e^{-j u t} d t \\
& A F_{(a, b, c, d)}(\tau, u)=\int_{-\infty}^{\infty} f\left(t+\frac{\tau}{2}\right) f^{*}\left(t-\frac{\tau}{2}\right) K_{A}(t, u) d t
\end{aligned}
$$

From Equations (36) and (37), the classical AF can be considered as the special case of AFL. The AFL has three additional freedoms, i.e., the parameters characterizing the LCT, which makes AFL more attractive for signal processing. In the AFL plane, we can separate the signal terms from cross term by adapting the parameters, the cross term suppression can also be achieved effectively with the AFL, which can be seen from the simulations.

In this sense, the RAFL can be looked as one generalization of the RAT, and we have more freedom by using the RAFL than the RAT in the non stationary signal detection and parameter estimation.

\section{The RAFL and the WVD-Hough transform (WHT)}

The Hough transform was proposed to fetch straight line at first, but was generalized to any graph later. The WVD combined with HT has been applied to the detection and parameter estimation of chirp signal, including multicomponent signals, in spite of the cross term produced by the WVD. The WHT can be interpreted as a line integral of the WVD in [17]: 


$$
\begin{aligned}
\operatorname{WHT}(f, g) & =\int_{-\infty}^{\infty} \int_{-\infty}^{-\infty} \operatorname{WVD}(t, v) \delta(v-f-g t) d t d v \\
& =\int_{-\infty}^{\infty} \operatorname{WVD}(t, f+g t) d t
\end{aligned}
$$

or equivalently, the WHT of a signal can be seen as the FT of its AF.

$$
\mathrm{WHT}(f, g)=\int_{-\infty}^{\infty} \operatorname{AF}(\tau, g \tau) e^{-j 2 \pi f \tau} d \tau
$$

In fact, it is easy to find that the WHT is equivalent to the RWT and RAT, so the RAFL can be seen as one generalization of the WHT. The WHT of a signal will generates peaks in the final domain, whose coordinates are the phase parameters $f_{0}$ and $g_{0}$. In the plane $(f, g)$, if one peak exceeds the threshold, the presence of a chirp is decided. The WHT is a good tool for the signal detection, even in the environment embedded in noise.

The WVD combined with HT provides a bridge between the signal and image processing. However, compared with AFL, the WHT requires a large computation.

\section{The FRFT}

The FRFT is actually a unified time-frequency transform. With the order from 0 increasing to 1 , the FRFT can reveal the characteristic of the signal gradually changing from the time domain to the frequency domain. Unlike usual quadratic timefrequency distributions, it reveals the time-frequency characteristics with a single variable, and does not suffer from cross-terms. Compared with the traditional FT, the FRFT does better in non-stationary signals processing especially in the chirp-like signals processing. Moreover, one extra degree of freedom (the order) may sometimes help to obtain better performance than the usual time-frequency distributions or the FT. And its developed fast algorithms lead to little computation load for good performance.

The FRFT of signal $f(t)$ is:

$$
F^{\alpha}[f(t)]=F_{f}(\alpha, u)=\int_{-\infty}^{\infty} K_{\alpha}(t, u) f(t) d t
$$

with

$$
K_{\alpha}(t, u)=\sqrt{\frac{1-\cot \alpha}{2 \pi}} e^{j\left(u^{2} / 2 \cot \alpha-u t \csc \alpha+t^{2} / 2 \cot \alpha\right)} \quad(\alpha \neq 0)
$$

As is known to us, there will be the maxima in the 2-D $F_{f}(\alpha, u)$, whose coordinate $(\alpha, u)$ corresponds to the initial frequency and chirp rate.

$$
\left\{\begin{array}{l}
m=-\cot \alpha \\
w_{0}=u / \sin \alpha
\end{array}\right.
$$

The RWT can be computed by dechirping:

$$
\operatorname{RWT}(u, \alpha)=\left|\frac{1}{|\sin \alpha|} \int_{-\infty}^{\infty} f(t) e^{-j\left(w_{0} t+m t^{2} / 2\right)} d t\right|_{\substack{m=-\cot \alpha \\ w_{0}=u / \sin \alpha}}^{2}
$$

And the square of FRFT:

$$
\left|F_{x}(\alpha, u)\right|^{2}=\left|\frac{1}{2 \pi \sin \alpha}\right|\left|\int_{-\infty}^{\infty} e^{j\left(-u t \csc \alpha+t^{2} / 2 \cot \alpha\right)} d t\right|^{2}
$$

From Equation (42) and Equation (43), we obtain

$$
\operatorname{RWT}(u, \alpha)=a \pi\left|F_{x}(\alpha, u)\right|^{2}
$$

That is to say, the FRFT is equivalent to RWT.

\section{Simulations}

In order to verify the derived results in this article and show the advantages of the AFL in the LFM signal detection, the simulations are performed in this section. From these simulations, it is easy to see that the AFL has a better performance in detection of the LFM signal than the traditional ones.

\section{Detection of LFM signal}

\section{Detection of one component LFM signal}

An one component LFM signa is defined as

$$
f(t)=\frac{1}{\sqrt{T}} e^{j\left(w_{0} t+\frac{m_{0}}{2} t^{2}\right)} \quad\left(|t|<\frac{T}{2}\right) .
$$

The parameters are chosen to be $T=40 \mathrm{~s}, w_{0}=0.1 \mathrm{rad} / \mathrm{s}$, $m_{0}=0.4 \mathrm{rad} / \mathrm{s}^{2}$. The $|\mathrm{AFL}|$ of $f(t)$ can be calculated as

$\left|A F_{(a, b, c, d)}(\tau, u)\right|= \begin{cases}\frac{\sin \left[\left(u / b-m_{0} \tau\right) T / 2\right]}{\left(u / b-m_{0} \tau\right) T / 2} \sqrt{\frac{1}{2 \pi b}} & (a=0) \\ \left|\sqrt{\frac{1}{2 \pi b}} \int_{-T / 2}^{T / 2} e^{j \frac{a}{2 b} t^{2}+j\left(m_{0} \tau-u / b\right) t} d t\right| & (a \neq 0)\end{cases}$

The detection of the single component LFM signal with $(a, b, c, d)=(0,2,-1 / 2,1)$ is performed. The contour part of $A F_{(a, b, c, d)}(\tau, u)$ and the detector $\eta(m)$ are plotted in Figure 1a,b, respectively.

It is clear from Figure 1a that the AFL passes through the origin of the AFL plane, and the slop of the projection line is $b * m_{0}$, which is the same as discussed before. Figure $1 \mathrm{~b}$ shows the value and upper bound of detector $\eta(m)$. There is one peak around the chirp rate $m_{0}$, as discussed in preceding sections. Therefore, we can detect the LFM signal based on RAFL.

\section{Detection of multicomponent LFM signal}

Suppose the $p$-component signal defined as following

$$
f(t)= \begin{cases}\sum_{i=0}^{p} \frac{1}{\sqrt{T}} e^{j\left(w_{i} t+\frac{m_{i}}{2} t^{2}\right)} & \left(|t|<\frac{T}{2}\right) \\ 0 & \left(|t|>\frac{T}{2}\right)\end{cases}
$$

Figure 2 plots the detector $\eta(m)$ of multicomponent LFM signal with $\mathrm{T}=40 \mathrm{~s}$ and different parameters. It can be concluded from Figure 2 that the detector can be used for detection of LFM signal with unequal amplitude.

Detection of multicomponent LFM signal The timefrequency analysis for multicomponent LFM signal will produce the cross term. Cross term suppression is an important problem in signal processing. Two ways have been proposed to solve the problem: kernel function filter and filter in the AF domain. The former is to design a kernel function according to some constraints derived by the goal, the later is to make use of 

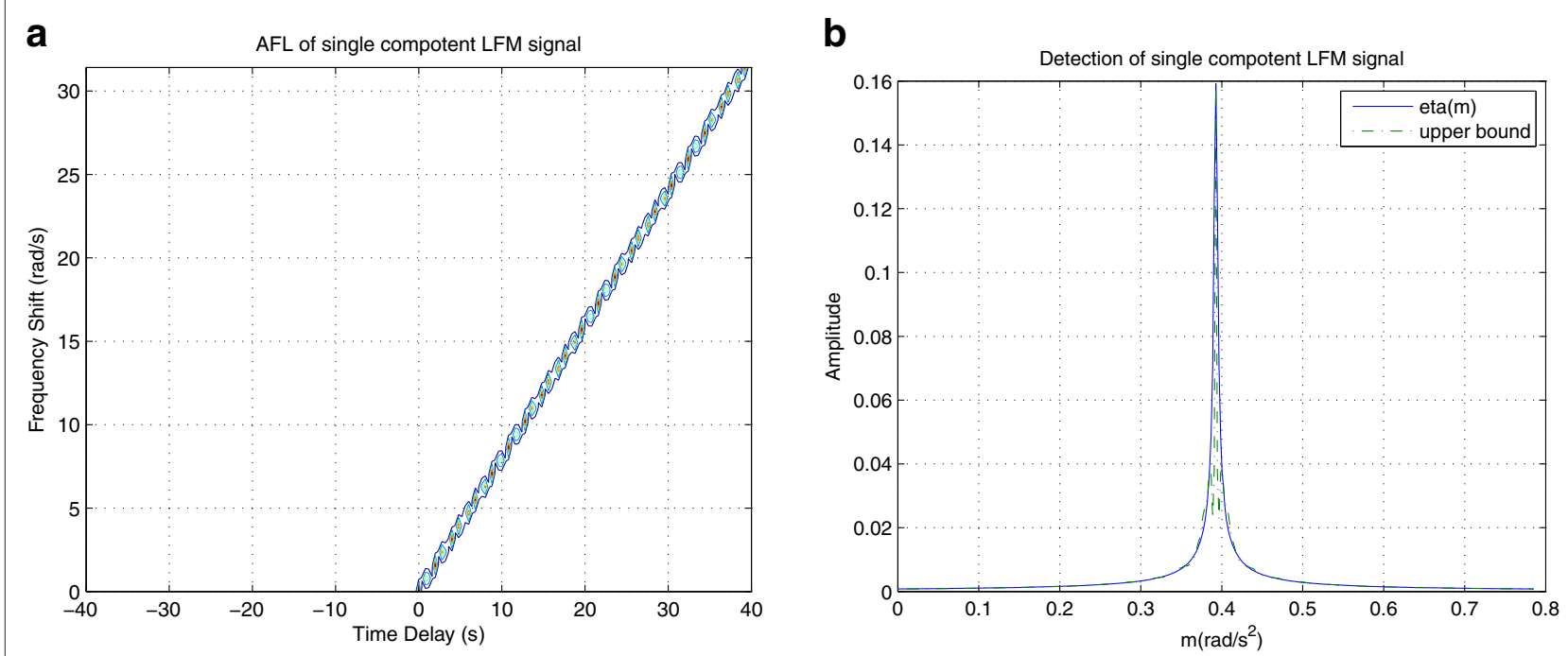

Figure 1 Detections of the single component LFM signal with $(a, b, c, d)=(0,2,-1 / 2,1)$.

the unique property of the AF. The AFL proposed in this article has three additional freedoms, i.e., the parameters characterizing the LCT, which will make the AFL more attractive for the analysis of signal and cross term suppression.

Figure 3 plots the detections results of the LFM signal with very close chirp rate with $\mathrm{T}=40 \mathrm{~s}$. Resolution, which is a common parameter for measuring the ability to separate two chirp signals. In this simulation, the resolution is defined as the minimum chirp rate separation to resolve two equally strong LFM signals in noise-free environment. Through computer simulations for trial signal with signal length $\mathrm{T}$ ranging from 10 to $60 \mathrm{~s}$, we obtain the resolution to be about $b / T^{2}$.
The Figure 3 shows the critically resolved cases of $\eta(m)$ for $\mathrm{T}=40 \mathrm{~s}$. It reveals that the resolution is proportional to the square of the signal length $T$. As discussed before, RAFL is one generalization of RAT, and the chirp rate resolution is consistent with RAT in [28], i.e., $1 / T^{2}$ level. The signal length must be greater than $\sqrt{b / \Delta}$ to achieve chirp rate resolution $\Delta$.

\section{Detection of LFM signal with noise}

The one component LFM signal with noise is defined as

$$
f(t)=\frac{1}{\sqrt{T}} e^{j\left(w_{0} t+\frac{m_{0}}{2} t^{2}\right)}+n(t) \quad\left(|t|<\frac{T}{2}\right)
$$
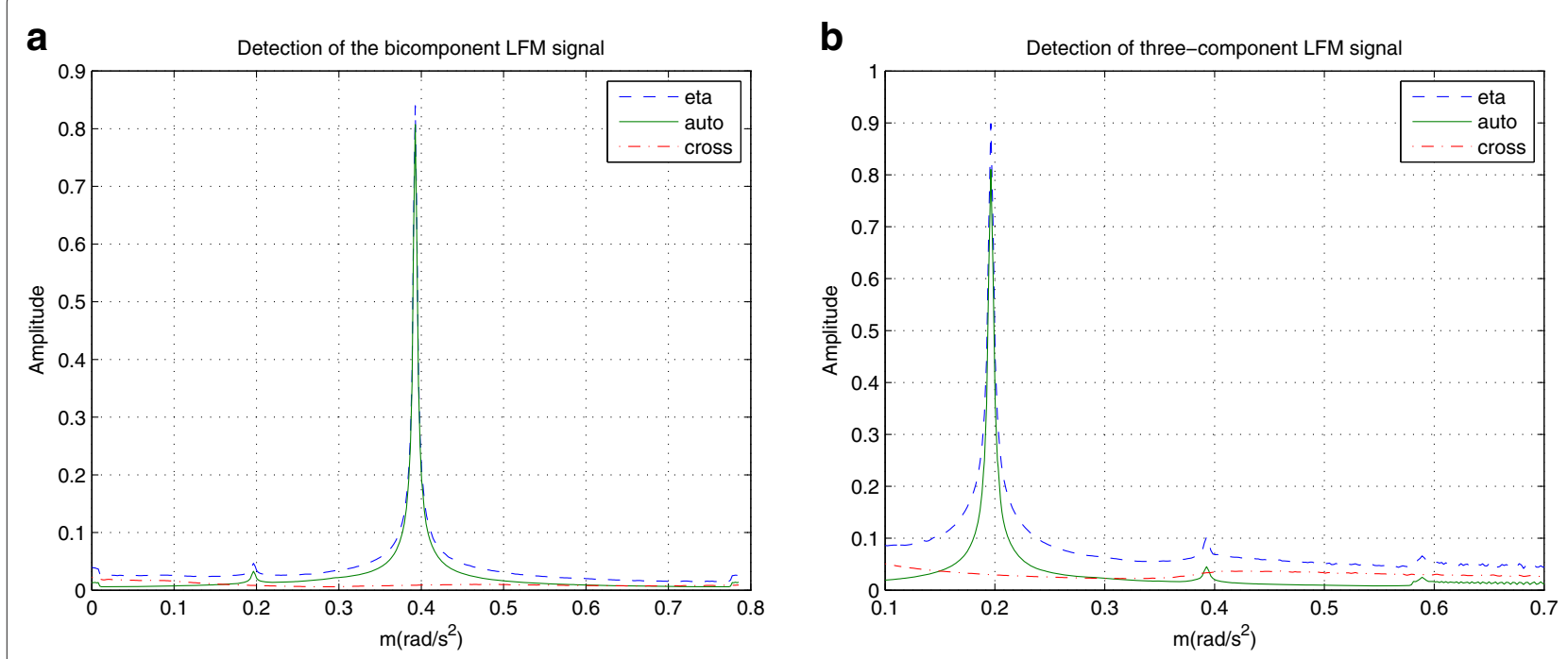

Figure 2 The detector $\eta(m)$ of multicomponent LFM signal with $T=40$ s and different parameters. (a) The $\eta(m)$ of the bicomponent LFM signal with amplitude of one component being one tenth of other. $(a, b, c, d)=(1 / 32,16,-1 / 32,16), w_{0}=w_{1}=0.1 \mathrm{rad} / \mathrm{s}, \mathrm{m}_{0}=0.4 \mathrm{rad} / \mathrm{s}^{2}, \mathrm{~m}_{1}=$ $0.2 \mathrm{rad} / \mathrm{s}^{2}$. (b) The $\eta(m)$ of three-component signal with different amplitude. $(a, b, c, d)=(1 / 32,32,-1 / 64,16), w_{0}=w_{1}=w_{2}=0.1 \mathrm{rad} / \mathrm{s}, m_{0}=$ $0.2 \mathrm{rad} / \mathrm{s}^{2}, m_{1}=0.4 \mathrm{rad} / \mathrm{s}^{2}, m_{2}=0.6 \mathrm{rad} / \mathrm{s}^{2}$. 

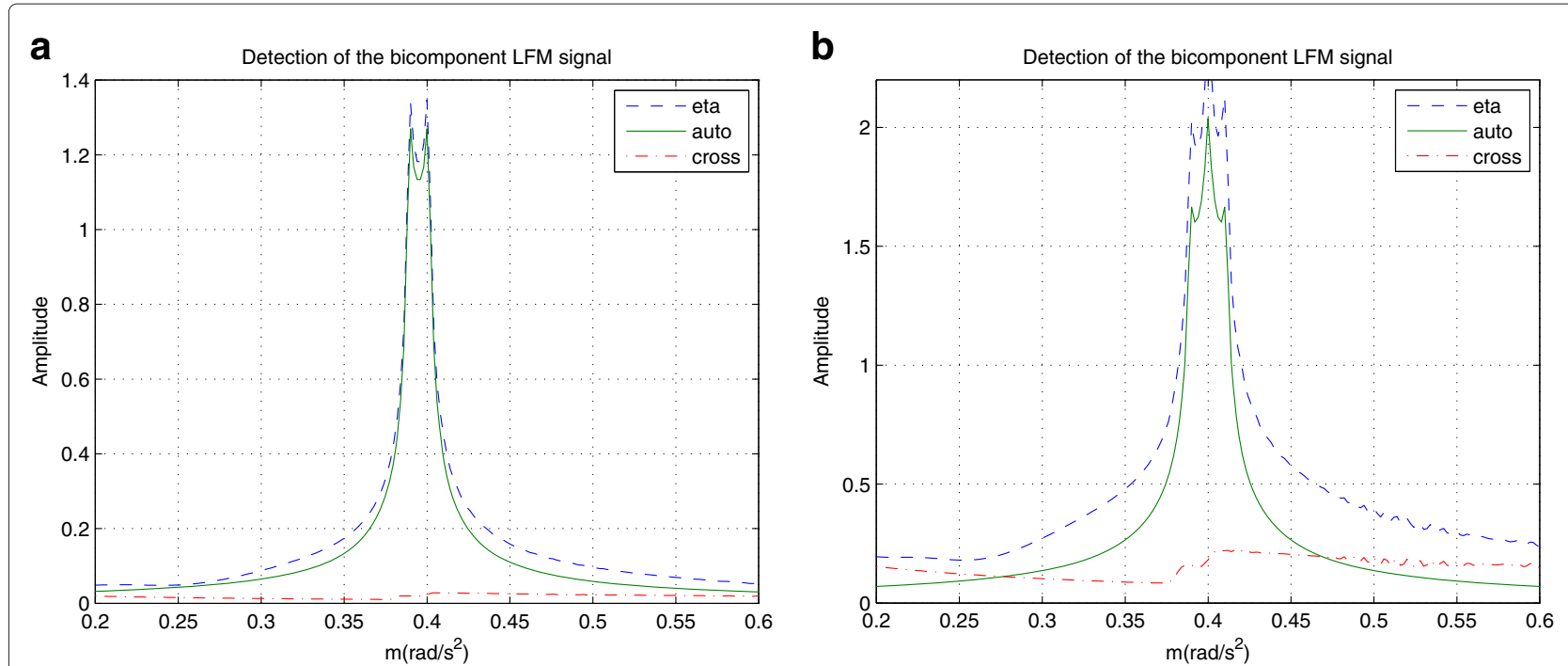

Figure 3 The detection results of the LFM signal with very close chirp rate. (a) $w_{0}=w_{1}=0.1 \mathrm{rad} / \mathrm{s}, \mathrm{m}_{0}=0.4 \mathrm{rad} / \mathrm{s}^{2}, \mathrm{~m}_{1}=0.39 \mathrm{rad} / \mathrm{s}^{2}$. (b) $w_{0}=w_{1}=w_{2}=0.1 \mathrm{rad} / \mathrm{s}, m_{0}=0.41 \mathrm{rad} / \mathrm{s}^{2}, m_{1}=0.4 \mathrm{rad} / \mathrm{s}^{2}, m_{2}=0.39 \mathrm{rad} / \mathrm{s}^{2}$.

where the noise $n(t)$ is assumed to be the white Gaussian noise with zero-mean, and variance $\sigma^{2}$.

Of course, the $p$-component LFM signal embedded in noise is defined as

$$
f(t)=\sum_{i=0}^{p} \frac{1}{\sqrt{T}} e^{j\left(w_{i} t+\frac{m_{i}}{2} t^{2}\right)}+n(t) \quad\left(|t|<\frac{T}{2}\right)
$$

The outputs of the detector are shown in Figure 4, with SNR's equal to $\infty,-10$, and $-20 \mathrm{~dB}$, respectively. It can be seen from Figure 4 that the detector shows the presence of the three component LFM signal under the strong noise background, with $m_{1}$ and $m_{2}$ being very close. These simulations have verified the effectiveness of the proposed detector.
Comparison the detectors of different time-frequency tools In this section, we will take finite-length LFM signal for instance to make the comparison of the detectors of different time-frequency tools. Figure 5 plots the comparison results among the common detectors for LFM signal in Equation (42) with $T=40 s, w_{0}=w_{1}=0.1 \mathrm{rad} / s, m_{0}=0.2 \mathrm{rad} / s^{2}, m_{1}=$ $0.4 \mathrm{rad} / s^{2}$.

It can be seen from Figure 5a that the RWT can detect the LFM signal for two peaks around the chirp rate. The RWT result is obtained by applying the RWT to the LFM signal and then compute the line integral along the straight line with its direction specified by the delta function $\delta\left(u-b m \tau-w_{0}\right)$.
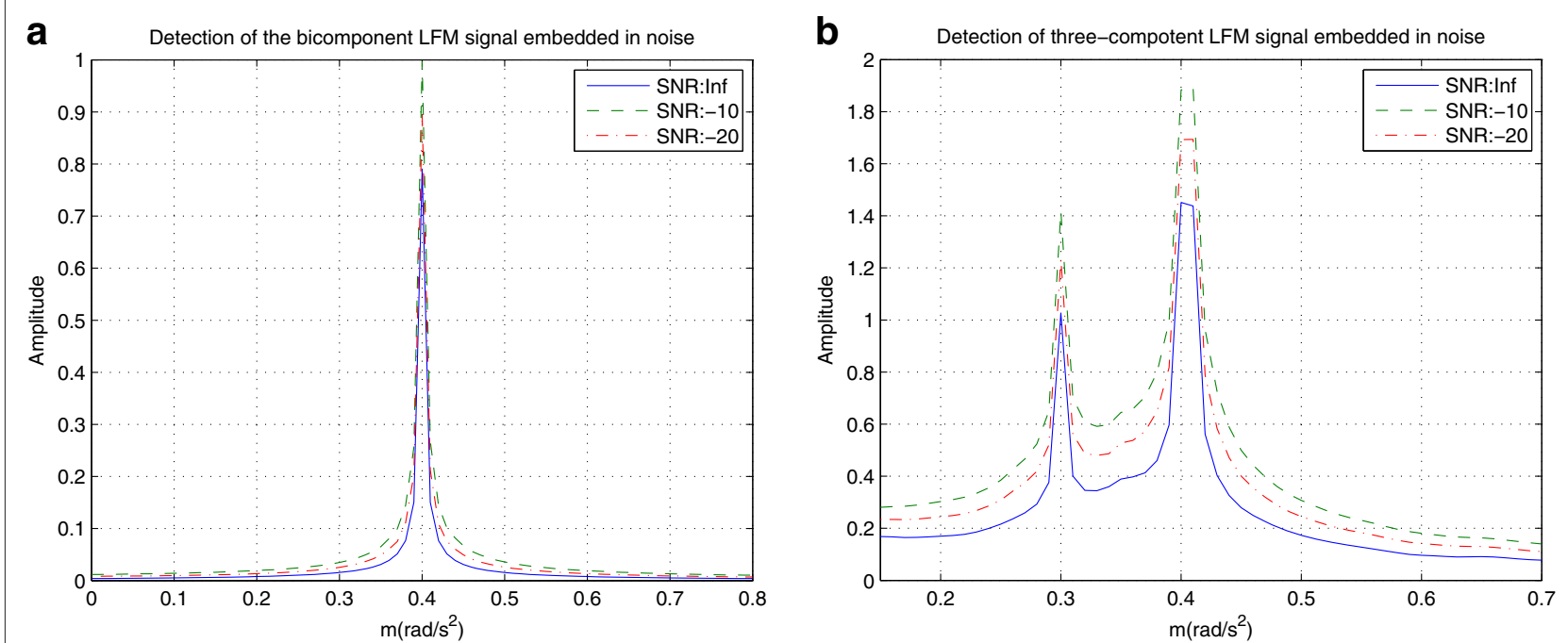

Figure 4 The detections of LFM signal embedded in Gaussian noise. (a) An one component LFM signal, with $w_{0}=0.1 \mathrm{rad} / \mathrm{s}, \mathrm{m}_{0}=0.4 \mathrm{rad} / \mathrm{s}^{2}$. (b) The three-component LFM signal, with $w_{0}=0.1 \mathrm{rad} / \mathrm{s}, m_{0}=0.3 \mathrm{rad} / \mathrm{s}^{2}, w_{1}=0.2 \mathrm{rad} / \mathrm{s}, m_{1}=0.4 \mathrm{rad} / \mathrm{s}^{2}, w_{2}=0.3 \mathrm{rad} / \mathrm{s}^{2}, \mathrm{~m}_{2}=0.41 \mathrm{rad} / \mathrm{s}^{2}$, $T=40$ s. 


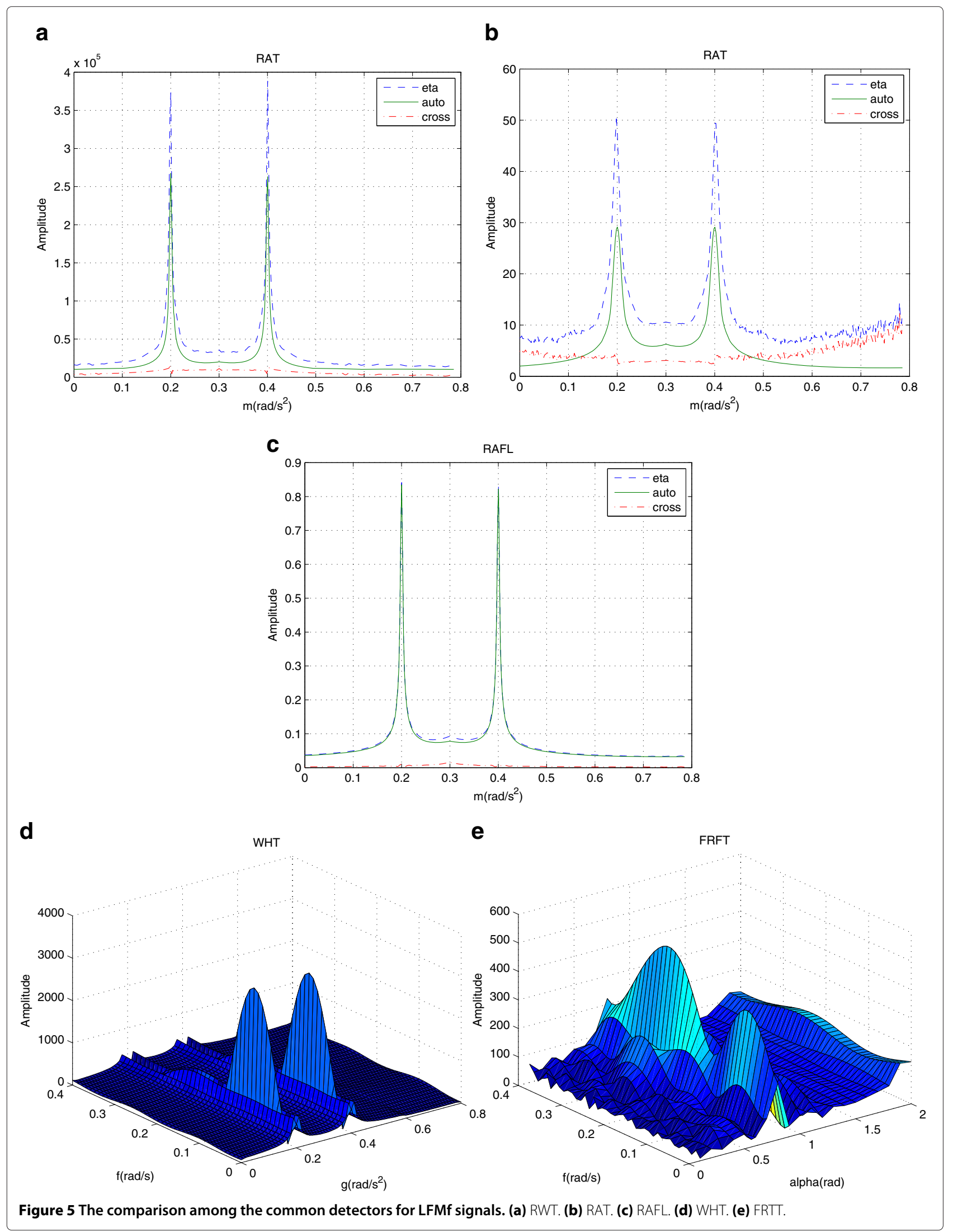


However, when $w_{0} \neq w_{1}$, the problem will be a 2 -D search problem.

We can see from Figure $5 \mathrm{~b}$ that the RAT is one of good time-frequency tools, however, the cross term is still serious, furthermore cross terms will interfere the auto terms. In Figure $5 \mathrm{~d}$, the WHT of the LFM signal can form peaks in the plane, and coordinates of the peak correspond to the parameters $w$ and $m$. But the computation is very large, and it is not so obvious to find the sharp peaks, which are the defects of WHT. The FRFT of the LFM signal displays the time-frequency focus at the special order. The detection and parameter estimation of LFM signal can be gained by 2-D searching in FRFT domain. However, there are some problems to solve, such as computation, etc. And the FRFT of multicomponent LFM signal with finite time may be not satisfactory, which can be seen from Figure 5e. Finally, it is easy to find from Figure $5 \mathrm{c}$ that the RAFL proposed in the manuscript can detect the multicomponent LFM signal, moreover the performance is 1-D search and effect is better.

The comparisons and descriptions above are only seen from the simulations. Now we can define a quantitative index to investigate the effectiveness of the detectors. As is known to us, the cross term suppression is an important problem for multicomponent LFM signal processing, so we define the quantitative index as

$$
r=\frac{|c r o|^{2}}{|t f|^{2}}
$$

where cro and $t f$ are the cross term value and time-frequency value, respectively. It represents the ratio of cross term relative to the overall value, which can be seen as the effectiveness of cross term suppression. The index of RWT, RAT and RAFL is $3.31,14.28,0.31 \%$, respectively. From the quantitative index, we can find that the RAFL proposed in this article can suppress the cross term effectively.

\section{Detection of QFM signal}

The QFM signal is defined as $f(t)=\frac{1}{\sqrt{T}} A e^{j\left(a_{1} t+a_{2} t^{2}+a_{3} t^{3}\right)}$ $\left(|t|<\frac{T}{2}\right)$, where $T=10, a_{1}=0.1, a_{2}=1, a_{3}=0.01$, the AFL of $f(t)$ is

$A F_{(a, b, c, d)}(\tau, u)=\left\{\begin{array}{l}\sqrt{\frac{2 \pi|b|}{j}} A^{2} e^{j\left(\frac{d}{2 b} u^{2}+a_{1} \tau+\frac{a_{3}}{4} \tau^{3}\right)} \delta\left(u-2 a_{2} \tau b\right) \\ \times\left(3 a_{3} \tau+\frac{a}{2 b}=0\right) \\ \sqrt{\frac{1}{6 a_{3} b \tau+a}} A^{2} e^{j\left(\frac{d}{2 b} u^{2}+a_{1} \tau+\frac{a_{3}}{4} \tau^{3}\right)-j \frac{\left(2 a_{2} \tau-u / b\right)^{2}}{2\left(6 a_{3} \tau+a / b\right)}} \\ \times\left(3 a_{3} \tau+\frac{a}{2 b} \neq 0\right)\end{array}\right.$

Figure 6 plots the AF and the AFL of this quadratic FM signal with $T=10 \mathrm{~s}, a_{1}=0.1 \mathrm{rad} / s, a_{2}=1 \mathrm{rad} / \mathrm{s}^{2}, a_{3}=0.01 \mathrm{rad} / \mathrm{s}^{3}$. We know from the Figure 6 a that the classical AF hardly detect the cubic phase. However, in Figure $6 \mathrm{~b}$ the performance of $a_{3}$ is more prominent.

In fact, the main information of the QFM signal is displayed by its cubic phase. That is to say, the cubic phase parameter can be seen as the information that we want to obtain. As the coefficient of the second phase is about 100 times of the cubic phase, it is can be considered that the SNR of $a_{3}$ is $-40 \mathrm{~dB}$ if the second phase is seen as noise relative to the cubic.

The AFL of QFM signal also pass through the origin of AFL plane. Therefore we use the same method to detect the QFM signal. Known from Equation (33), for power signal, if we choose the special parameter, AFL can generate one impulse, and for the energy signal with finite time, there will be the crest for energy accumulation.
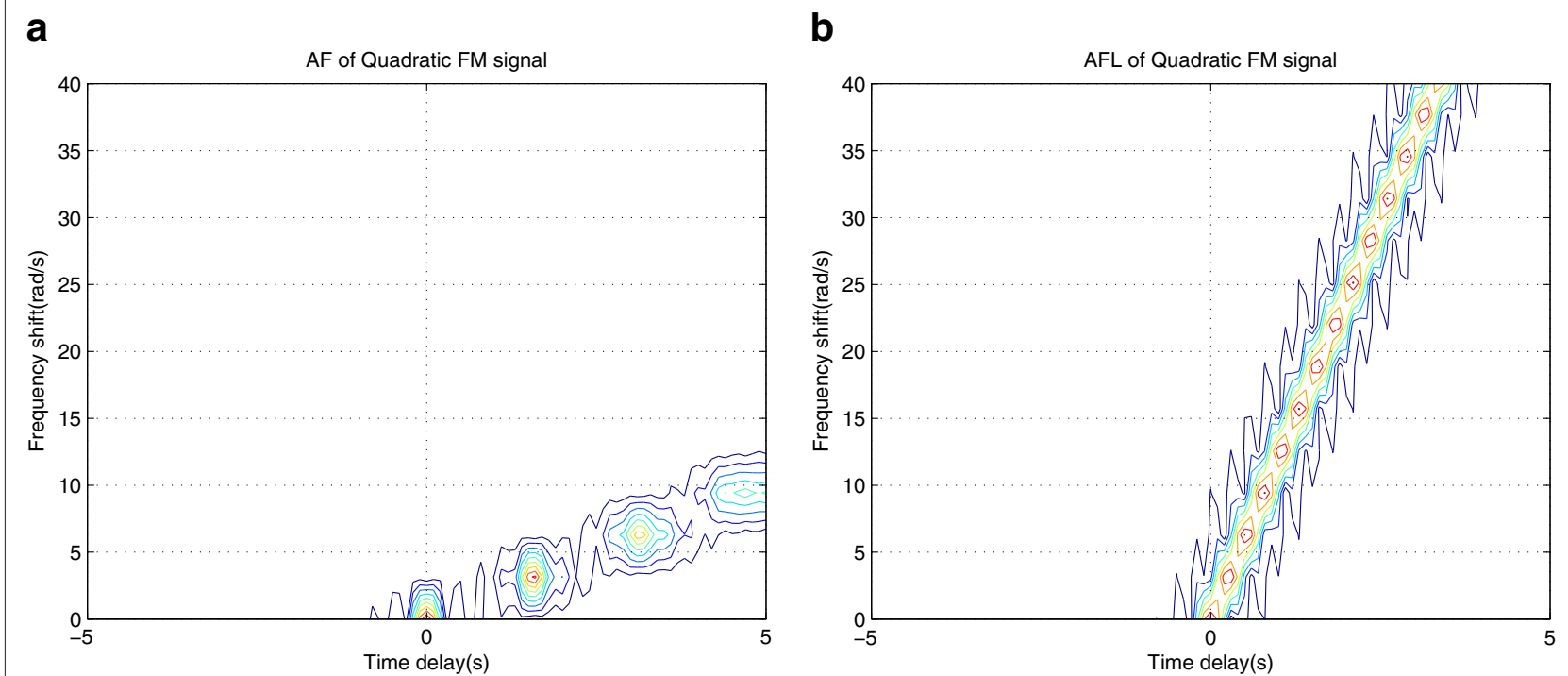

Figure 6 The AF and AFL of a quadratic FM signal with $T=10 \mathrm{~s}$. (a) AF.

(b) $\operatorname{AFL}$ with $(a, b, c, d)=(1,6,1 / 2,4)$. 


\section{Conclusion}

On the basis of the classical AF and local transform theory, this article proposes a new kind of definition of AF associated with the LCT (we name it as AFL), which can be seen as one of the generalizations of the classical AF. The main properties of AFL are investigated in detail. The AFL has three additional freedoms, i.e., the parameters characterizing the LCT, which makes the AFL more attractive for the analysis of LFM signal. The RAFL obtained by combining AFL with RT is applied to detect the LFM signal. It can generate the sharp peak around the chirp rate in RAFL plane, and the cross-term can be suppressed effectively, which can be verified from the simulation and comparison results. The future works along this direction is to explore the applications of the AFL in the non-stationary signal processing, and the comparison of the AFL with the other kind of AF proposed in $[29,30]$ in the LFM signal detection and the parameter estimation.

\section{Methods}

All of the signals used in the manuscript are produced by Matlab software, and the algorithm in the simulation section are proposed based on the main results proposed in Section "The applications of the AFL".

\section{Competing interests}

The authors declare that they have no competing interests.

\section{Acknowledgements \\ The authors would like to thank the handling editor and the anonymous reviewers for their valuable comments and suggestions. It helps us a lot in improving the manuscript significantly. The authors would also like to thank Dr. Hai Jin of the Beijing Institute of Technology for many discussions and the proofreading of the manuscript. This work was supported by the National Natural Science Foundation of China (No. 60901058 and No. 61171195) and also supported partially by Beijing Natural Science Foundation (No. 1102029)}

Received: 14 November 2011 Accepted: 27 May 2012

Published: 11 July 2012

\section{References}

1. L Cohen, Time-frequency distribution-a review. Proc. IEEE. 77(7), 941-981 (1989)

2. I Daubechies, Ten Lectures on Wavelets. (Philadelpha, PA: SIAM, 1992)

3. HM Ozaktas, MA Kutay, Z Zalevsky, The Fractional Fourier Transform with Applications in Optics and Signal Processing. (New York: Wiley, 2001)

4. R Tao, B Deng, Y Wang, Fractional Fourier Transform and its Applications. (Beijing: Tsinghua Univ. Press, 2009)

5. V Namias, The fractional order Fourier transform and its application to quantum mechanics. IMA J. Appl. Math. 25(3), 241-265 (1980)

6. AC McBride, FH Kerr, On Namias's fractional Fourier transforms. IMA J. Appl. Math. 39(2), 59-175 (1987)

7. LB Almeida, The fractional Fourier transform and time-frequency representation. IEEE Trans. Signal Process. 42, 3084-3091 (1994)

8. B Santhanam, JH McClellan, The discrete rotational Fourier transform. IEEE Trans. Signal Process. 44(4), 994-998 (1996)

9. HM Ozaktas, O Ankan, MA Kutay, G Bozdaki, Digital computation of the fractional Fourier transform. IEEE Trans. Signal Process. 44(9), 2141-2150 (1996)

10. S-C Pei, J-J Ding, Closed-form discrete fractional and affine Fourier transforms. IEEE Trans. Signal Process. 48(5), 1338-1353 (2000)
11. C-P Li, B-Z Li, T-Z Xu, Approximating bandlimited signals associated with the LCT domain from nonuniform samples at unknown locations. Signal Process. 92(7), 1658-1664 (2012)

12. R Tao, B-Z Li, Y Wang, On sampling of bandlimited signals associated with the linear canonical transform. IEEE Trans. Signal Process. 56(11), 5454-5464 (2008)

13. S-C Pei, J-J Ding, Eigenfunctions of linear canonical transform. IEEE Trans. Signal Process. 50(1), 11-26 (2002)

14. D Bing, TRan, W Yue, Convolution theorems for the linear canonical transform and their applications. Sci. China-Inf. Sci. 49(4), 592-603 (2006)

15. D Wei, Y Li, A convolution product theorem for the linear canonical transform. IEEE Signal Process. Lett. 16(10), 853-856 (2009)

16. B-Z Li, T-Z Xu, Spectral analysis of sampled signals in the linear canonical transform domain. Math. Probl. Eng. 2012, 19 (2012)

17. FS Oktem, HM Ozaktas, Exact relation between continuous and discrete linear canonical transforms. IEEE Signal Process. Lett. 16(8), 727-730 (2009)

18. A Koc, HM Ozaktas, C Candan, MA Kutay, Digital computation of linears canonical transforms. IEEE Trans. Signal Process. 56(6), 2383-2394 (2008)

19. G Kutyniok, Ambiguity functions, Wigner distributions and Cohen's class for LCA groups. J. Math. Anal. Appl. 277(2), 589-608 (2003)

20. RG Shenoy, TW Parks, Wide-band ambiguity functions and affine Wigner distributions. Signal Process. 41(3), 339-363 (1995)

21. HT Li, PM Djuric, MMSE estimation of nonlinear parameters of multiple linear/quadratic chirps. IEEE Trans. Signal Process. 46(3), 796-801 (1998)

22. CD Luigi, E Moreau, An iterative algorithm for estimation of linear frequency modulated signal parameters. IEEE Signal Process. Lett. 9(4), 127-129 (2002)

23. M Mboup, T Adalai, A generalization of the Fourier transform and its application to spectral analysis of chirp-like signals. Appl. Comput. Harmon. Anal. 32(2), 305-312 (2012)

24. C Zhe, W Hongyu, Q Tianshuang, Research of ambiguity function associated with the fractional Fourier trandform (in Chinese). Signal Process. 19(6), 499-502 (2003)

25. S Barbarossa, Analysis of multicomponent LFM signals by a combined Winger-Hough transform. IEEE Trans. Signal Process. 43(6), 1511-1515 (1995)

26. X-G Xia, Discrete chirp-Fourier transform and its application to chirp rate estimation. IEEE Trans. Signal Process. 48(11), 3122-3133 (2006)

27. XM LV, M Xing, Z Zhang, Z Bao, Keystone transformation of the Wigner-Ville distribution for analysis of multicomponent LFM signals. Signal Process. 89(5), 791-806 (2009)

28. M Wang, AK Chan, CK Chui, Linear frequency-modulated signal detection using Radon-Ambiguity transform. IEEE Trans. Signal Process. 46(3), 571-587 (1998)

29. S-C Pei, J-J Ding, Relations between fractional operations and time-frequency distributions and their applications. IEEE Trans. Signal Process. 49(8), 1638-1655 (2001)

30. H Zhao, Q-W Ran, J MA, L-Y Tan, Linear canonical ambiguity function and linear canonical transform moments. Optik. 122(6), 540-543 (2011)

31. JJ Healy, JT Sheridan, Cases where the linear canonical transform of a signal has compact support or is band-limited. Opt. Lett. 33(3), 228-230 (2008)

32. C Candan, HM Ozaktas, Sampling and series expansion theorems for fractional Fourier and other transforms. Signal Process. 83(11), 2455-2457 (2003)

33. B-Z Li, R Tao, Y Wang, New sampling formulae related to linear canonical transform. Signal Process. 87(5), 983-990 (2007)

34. JJ Healy, JT Sheridan, Sampling and discretization of the linear canonical transform. Signal Process. 89(4), 641-648 (2009)

35. J Zhao, R Tao, Y-L Li, Y Wang, Uncertainty principles for linear canonical transform. IEEE Trans. Signal Process. 57(7), 2856-2858 (2009) 
36. KK Sharma, SD Joshi, Uncertainty principles for real signals in linear canonical transform domains. IEEE Trans. Signal Process. 56(7), 2677-2683 (2008)

37. A Stern, Uncertainty principles in linear canonical transformdomains and some of their implications in optics. J. Opt. Soc. Am. 25(3), 647-652 (2008)

38. I Gradshteyn, I Ryzhik, Table of Integrals, Series, and Products. (San Diego, CA: Academic, 1980)

doi:10.1186/1687-6180-2012-138

Cite this article as: Che et al.: The ambiguity function associated with the linear canonical transform. EURASIP Journal on Advances in Signal Processing 2012 2012:138

\section{Submit your manuscript to a SpringerOpen ${ }^{\mathcal{O}}$ journal and benefit from:}

- Convenient online submission

- Rigorous peer review

- Immediate publication on acceptance

- Open access: articles freely available online

- High visibility within the field

- Retaining the copyright to your article

Submit your next manuscript at $\gg$ springeropen.com 